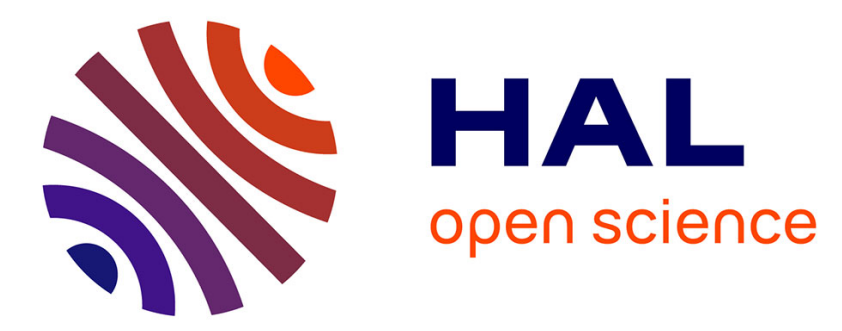

\title{
Model-based Feature Augmentation for Cardiac Ablation Target Learning from Images
}

Rocio Cabrera Lozoya, Benjamin Berte, Hubert Cochet, Pierre Jaïs, Nicholas Ayache, Maxime Sermesant

\section{- To cite this version:}

Rocio Cabrera Lozoya, Benjamin Berte, Hubert Cochet, Pierre Jaïs, Nicholas Ayache, et al.. Modelbased Feature Augmentation for Cardiac Ablation Target Learning from Images. IEEE Transactions on Biomedical Engineering, 2018, pp.1. 10.1109/TBME.2018.2818300 . hal-01744142

HAL Id: hal-01744142

https://hal.inria.fr/hal-01744142

Submitted on 7 May 2018

HAL is a multi-disciplinary open access archive for the deposit and dissemination of scientific research documents, whether they are published or not. The documents may come from teaching and research institutions in France or abroad, or from public or private research centers.
L'archive ouverte pluridisciplinaire HAL, est destinée au dépôt et à la diffusion de documents scientifiques de niveau recherche, publiés ou non, émanant des établissements d'enseignement et de recherche français ou étrangers, des laboratoires publics ou privés. 


\title{
Model-based Feature Augmentation for Cardiac Ablation Target Learning from Images
}

\author{
Rocío Cabrera Lozoya ${ }^{1,2}$, Benjamin Berte ${ }^{2}$, Hubert Cochet $^{2}$, Pierre Jaïs ${ }^{2}$, Nicholas Ayache ${ }^{1}$, Maxime Sermesant ${ }^{1}$
}

\begin{abstract}
Goal: We present a model-based feature augmentation scheme to improve the performance of a learning algorithm for the detection of cardiac radio-frequency ablation (RFA) targets with respect to learning from images alone. Methods: Initially, we compute image features from delayed-enhanced MRI (DE-MRI) to describe local tissue heterogeneities and feed them into a machine learning framework with uncertainty assessment for the identification of potential ablation targets. Next, we introduce the use of a patient-specific image-based model derived from DE-MRI coupled with the Mitchell-Schaeffer electrophysiology model and a dipole formulation for the simulation of intracardiac electrograms (EGM). Relevant features are extracted from these simulated signals which serve as a feature augmentation scheme for the learning algorithm. We assess the classifier's performance when using only image features and with model-based feature augmentation. Results: We obtained average classification scores of $97.2 \%$ accuracy, $\mathbf{8 2 . 4} \%$ sensitivity and $\mathbf{9 5 . 0} \%$ positive predictive value (PPV) by using a model-based feature augmentation scheme. Preliminary results also show that training the algorithm on the closest patient from the database, instead of using all the patients, improves the classification results. Conclusion: We presented a feature augmentation scheme based on biophysical cardiac electrophysiology modeling to increase the prediction scores of a machine learning framework for the RFA target prediction. Significance: The results derived from this study are a proof of concept that the use of model-based feature augmentation strengthens the performance of a purely image driven learning scheme for the prediction of cardiac ablation targets.
\end{abstract}

Index Terms-radio-frequency ablation planning, cardiac electrophysiology modelling, intracardiac electrogram modelling, electroanatomical mapping

\section{INTRODUCTION}

Cardiovascular diseases (CVD) remain, to date, the leading cause of death in the western world. According to the Global Burden of Disease, CVD were responsible for more than $29 \%$ of deaths in the world in 2013 (> 15,616 million deaths), twice the amount of deaths caused by cancer in the same year [1]. Cardiac arrhythmias are a subset of CVD grouping abnormalities in the heart rhythm. A dangerous consequence of these rhythm perturbations includes a compromise of the heart's effectiveness to pump blood. Sudden cardiac death (SCD) occurs if the condition is not treated within a very

Copyright (c) 2010 IEEE. Personal use of this material is permitted. However, permission to use this material for any other purposes must be obtained from the IEEE by sending a request to pubs-permissions@ieee.org. rocio.cabrera_lozoya@inria.fr - Tel: +33492387660 - 2004 route des Lucioles BP-93, 06902 Sophia-Antipolis Cedex, FRANCE

1 Inria Sophia-Antipolis, Asclepios Research Group, Sophia-Antipolis, France

2 IHU Liryc, Hôpital Cardiologique du Haut-Lévêque, Bordeaux, France

Manuscript received November X, 2016. short delay. Early detection and accurate prediction of disease progression of cardiac arrhythmias remain an important need to reduce their mortality. Furthermore, improvements in therapy planning and guidance are of vital importance to reduce the mortality of these diseases.

A potentially curative treatment for cardiac arrhythmias is radiofrequency ablation (RFA), where thermal lesions are generated in the heart to interrupt abnormal re-entry circuits that cause arrhythmias. The greatest challenge in this therapy is ablation target identification. To date, there exists no universal consensus on the optimal ablation strategy, but a number of techniques can be found in the literature [2]. Some report the use of line lesions going from the regions of dense scar to those of normal myocardium [3] or that transected all potential isthmuses [4]. Other approaches focus on the study of reentry isthmuses [5], [6] enhanced through the use of complementary imaging modalities, such as MRI or PET [7], [8] while other strategies include the assessment of pace maps [9], [10].

Electrocardiographic imaging (ECGI) is a relatively new, noninvasive imaging modality that reconstructs potentials, electrograms, and isochrones on the epicardial surface from body surface measurements [11]. The work by $\mathrm{Li}$ and $\mathrm{He}$ [12] solves this inverse problem by means of heart-model parameters (onset activation location) and was further extended [13] and validated on rabbits [14] and swines [15], [16]. More recently, [17] was able to reconstruct re-entry circuits to correctly reveal both epicardial and endocardial origins of activation, consistent with locations of exit sites confirmed from the ablation procedure. Nevertheless, while it is useful in capturing and analysing global activation patterns, it still has limitations to assess the local abnormal ventricular activities (LAVA) like those studied here which serve as ablation targets for cardiac arrhythmia treatment.

The study in [18] analysed rates of ventricular tachycardia (VT) recurrence in patients undergoing ablation limited to clinical VT along with mappable VTs versus substrate-based ablation. The first subset of patients underwent conventional mapping techniques to define the mechanism of the arrhythmias and identify potential sites for ablation. After complete substrate mapping, pacing protocols were used to induce clinical VT. Linear ablation lesions were placed to transect the VT isthmus and terminate inducible VTs [18]. In the subset of patients undergoing substrate-based ablation, careful identification of fractionated, delayed, or abnormal electrograms was performed to eliminate all abnormal potentials. The study found that an extensive substrate-based ablation approach is superior to ablation targeting only clinical and stable VTs in patients with ischemic cardiomyopathy presenting with 


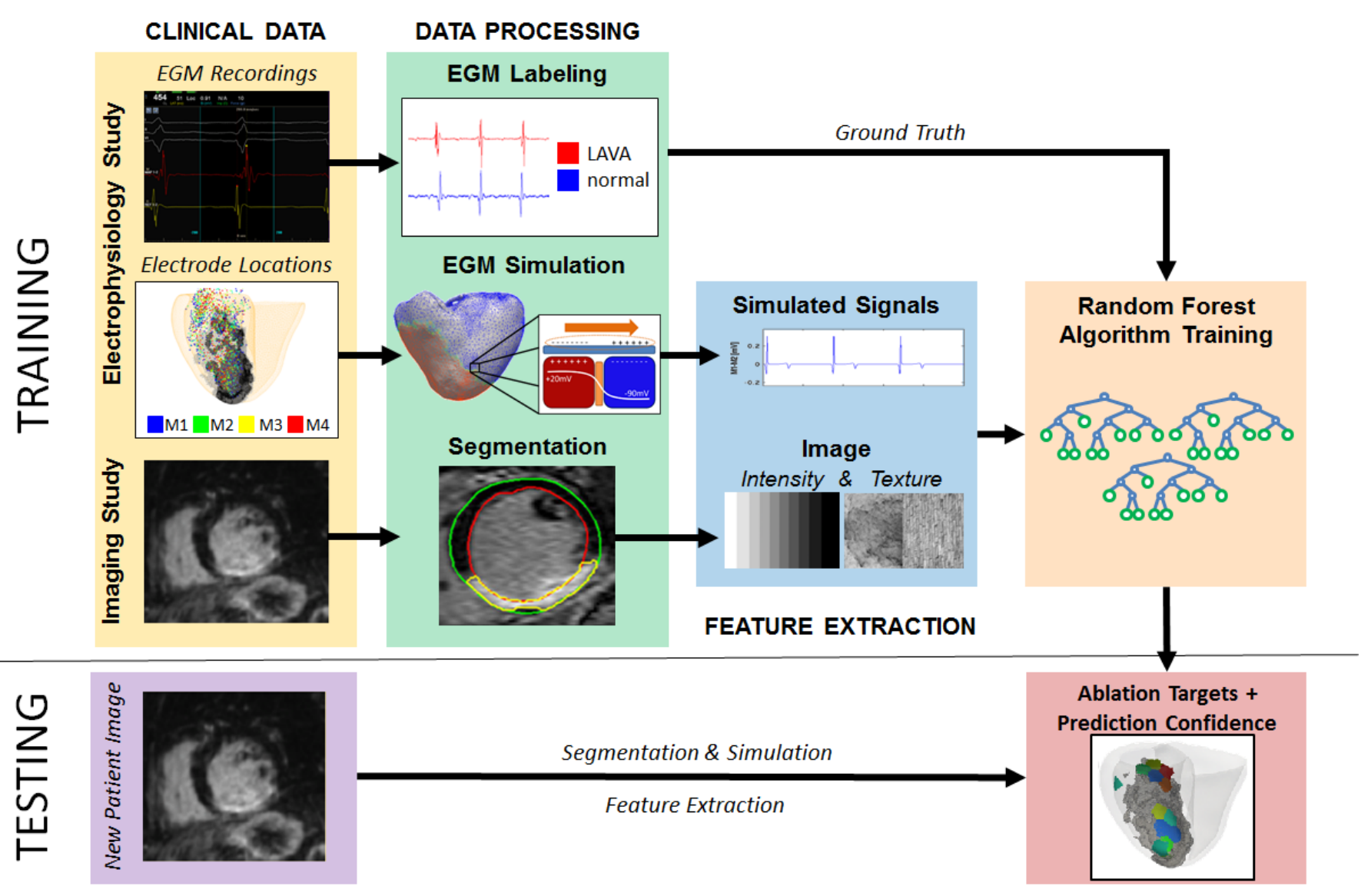

Fig. 1: General processing pipeline showing the clinical data and the data processing, feature extraction and learning stages. It shows EGM labels from clinical data being used only on the training process. Electrode locations (from the clinical EP study) are used as reference points where the simulated electrograms are generated and where MR image features are computed. Both simulated and image features are fed into the random forest algorithm for training. Finally, the testing stage consists of obtaining a newly seen dataset and computing the corresponding features which are fed to the trained algorithm to obtain prediction labels with a certain confidence level.

tolerated VT [18].

We will focus our attention on a recent study with a cohort of 70 patients suffering from VT and structurally abnormal ventricles which proposed the elimination of LAVA as an endpoint for RFA therapy and concluded that it was associated with a reduction in recurrent VT or death during long-term follow-up [19].

LAVA identification can be achieved using electrophysiological (EP) substrate mapping, however this is a lengthy procedure ( $>6$ hours), requiring a significant amount of $\mathrm{X}$ ray imaging and an expert electrophysiologist's knowledge to appropriately identify all LAVA instances. There is still a high rate of recurrences due to missed ablation targets, as their identification of LAVA is still a challenge.

It has been hypothesized that the generation of these abnormal electrical activities is related to regions with the presence of surviving myocardial fibers within scar tissue. Delayedenhanced magnetic resonance imaging (DE-MRI) remains, to date, the gold standard for myocardial size and morphology evaluation and for assessment of the heterogeneity of the border zone [20]. In our context, it is useful to non-invasively assess scar topology and tissue heterogeneity. Areas of intermediate signal intensity in DE-MRI, referred to as the grey zone, are likely to host both scarred and surviving myocardium related to arrhythmia in ischemic populations [19] [21].

In previous works [22], we demonstrated the feasibility in the identification of RFA targets using only DE-MR imagebased features in a machine learning framework while integrating the influence of the inherent error sources of the complex multi-modal data in the training set. Furthermore, we presented in [23] the use of patient-specific anatomical models constructed from DE-MR images coupled with a biophysical electrophysiology model for the synthetic simulation intracardiac electrograms with distinguishable healthy and LAVA-like characteristics.

We build up on our previous publication on signal simulation [23] in order to show that model-based feature augmentation can introduce physiological knowledge from modelling into the data-driven capabilities of machine learning. The contributions of this manuscript are:

- The combined use of imaging and modelling to improve the identification of ablation targets 
- An uncertainty assessment inspired from the works on cost-sensitive learning to account for the impact of noisy or inaccurate ground truth when being fed into learning algorithms. A preliminary version of this work was presented in [23].

- The analysis of the relative impact of using only imaging data or imaging data together with model-based feature augmentation on classification performance.

- The application of our full pipeline on a clinical patient cohort consisting of five subjects whose ground truth was constructed and revised by experienced clinicians.

The pipeline of our work is shown in Figure 1 and the structure of this paper is as follows: we first present the acquisition and processing stages of the clinical data used for our experiments. We then describe the feature extraction process for the imaging data. The framework used for the simulation of intracardiac EGM is then explained, as well as the features of the resulting signals. Then, we include an overview of the uncertainty assessment followed by the evaluation metrics used to assess the classification performance. Finally, the results obtained through the use of only image features and image features augmented with model-based features are presented and their relevance is discussed.

The results derived from this study open up possibilities for the inclusion of physiological knowledge into learning algorithms through feature augmentation schemes for noninvasive cardiac arrhythmia ablation planning. The clinical significance of this work stems from the possibility of incorporating modelling and machine learning techniques into a clinical workflow which would require only non-invasive, pre-intervention data in order to improve the accuracy of RFA target identification, therefore reducing intervention time and increasing the success rates of RFA. To the best of our knowledge, this is the first work presenting combined imagebased and model-based features in such a learning framework, and using it to predict ablation targets from non-invasive clinical data.

\section{Clinical Data}

Five patients referred for cardiac ablation for post-infarction ventricular tachycardia were included in this study. The patients underwent cardiac MRI prior to high-density EP contact mapping of the endocardium or epicardium. While the final aim of this work is to predict ablation targets from noninvasive clinical data, the invasive EP study is required as it will serve to obtain the ground truth necessary to train and validate our algorithm. The following section describes in more details the clinical data acquired.

\section{A. Imaging Data}

The scar tissue was imaged on a 1.5T MRI device (Avanto, Siemens Medical Systems) 15 minutes after the injection of a gadolinium contrast agent. A whole heart image was acquired using an inversion-recovery prepared, ECG-gated, respiratorynavigated, 3D gradient-echo pulse sequence with fat-saturation $\left(1.25 \times 1.25 \times 2.5 \mathrm{~mm}^{3}\right)$.
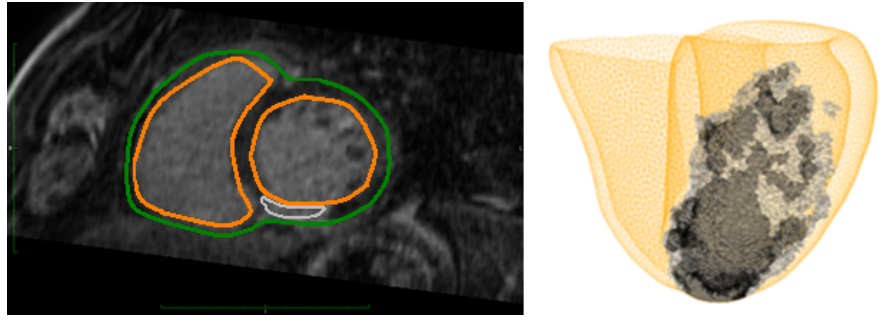

Fig. 2: (Left) DE-MRI slice with segmentation of the epicardium (green), endocardium (orange) and the scar (white) region. (Right) Anatomically personalised heart model denoting the healthy (yellow), grey-zone (light grey) and scarred (dark gray) tissues obtained from DE-MRI segmentations.
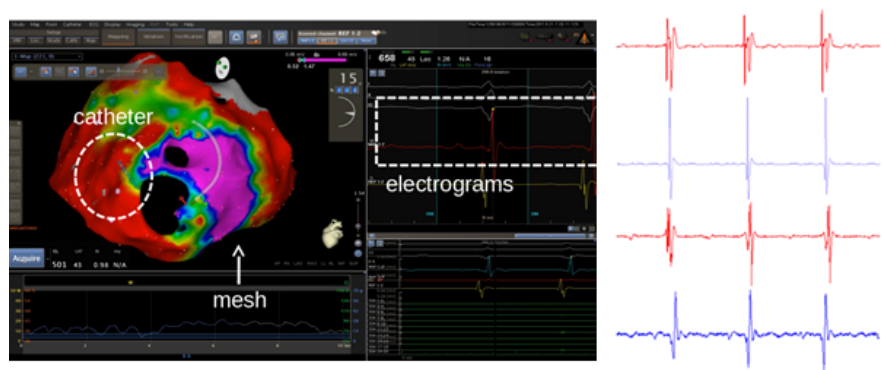

Fig. 3: (Left) CARTO mapping framework including catheter location, generated mesh and recorded electrograms. (Right) Samples of intracardiac electrograms labeled as LAVA (red) or non-LAVA (blue) by an experienced electrophysiologist. The top signal presents sharp potentials occurring after the QRS complexes which have a slightly higher frequency than the far-field ventricular potential, whereas the third signal presents fractionation during the first recorded beat, therefore complying with the definitions of LAVA. The second and third signals show normal depolarization patterns, nonetheless, the last signal presents a higher degree of noise in the baseline. Experienced electrophysiologists are trained in order to be able to detect LAVA even within signals with a certain degree of noise. Furthermore, if the recording was deemed to noisy for classification, it was discarded.

The epicardial and endocardial walls were manually segmented on reformatted images of isotropic voxel size $\left(0.625 \mathrm{~mm}^{3}\right)$ as shown on the left image in Figure 2. Abnormal myocardium (dense scar and grey zone areas) was segmented using adaptive thresholding of the histogram, with a cutoff at $35 \%$ of maximal signal intensity. Segmentations were reviewed by an experienced radiologist, with the option of manual correction, and allowed for the creation of anatomically personalised heart models as the one shown on the right image in Figure 2.

\section{B. Electrophysiological Data}

Electroanatomical mapping (EAM) is a minimally-invasive technique used to record in-vivo cardiac electrical activity at specific locations inside the heart. When the catheter comes in contact with the tissue of interest, both electrogram characteristics (i.e. activation time or voltage) of the tissue and the 
TABLE I: Electrophysiology Study Statistics

\begin{tabular}{ccccccc} 
Patient & \# Electrode Locations & \# M1-M2 EGMs & \# LAVA Samples & \# Non-LAVA Samples & \% LAVA in Dataset & Ratio Scar / Healthy Tissue \\
\hline 1 & 1472 & 368 & 71 & 297 & $19.3 \%$ & $3.7 \%$ \\
2 & 4804 & 1201 & 44 & 1157 & $24.2 \%$ & 0.25 \\
3 & 1356 & 339 & 82 & 17 & $20.6 \%$ & 0.08 \\
4 & 856 & 214 & 33 & 667 & $4.7 \%$ & 0.10 \\
5 & 2800 & 700 & & & 170
\end{tabular}

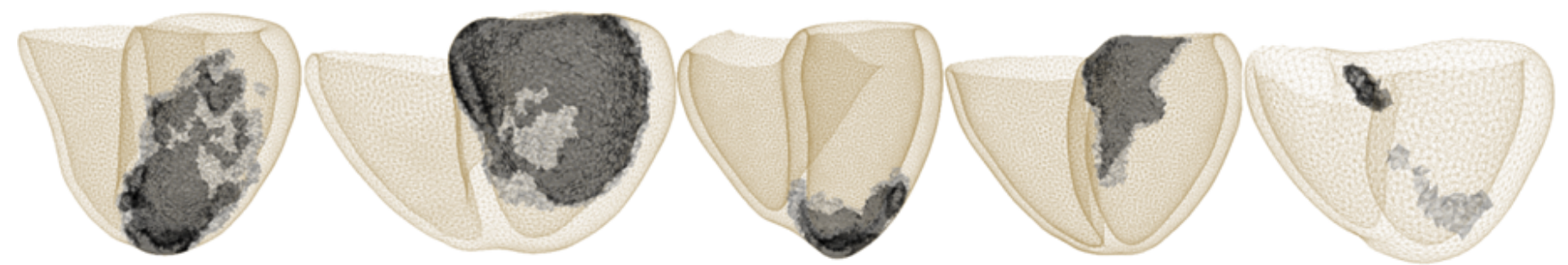

Fig. 4: Anatomically personalised heart models for Patients 1 to 5 showing healthy (brown), scar (black) and border zone (grey) tissues.

coordinates of the catheter's position in space through nonfluoroscopic localization are retrieved [2].

Despite the capability of locating the catheter in 3D space and therefore reconstructing the heart chamber that is being mapped, geometries provided by EAM systems tend to be rough estimates of the actual cardiac anatomy. Catheter position is highly affected by cardiac or respiratory motion, and anatomic reconstruction algorithms may vary between systems [2]. These limitations can compromise the integration of EAM information with anatomical information from traditional imaging systems.

For our study, the CARTO mapping system (Biosense Webster) is used. Contact mapping was achieved in sinus rhythm on the endocardium (trans-septal approach) with a dedicated multipolar mapping catheter (PentaRay, Biosense Webster). Signals were annotated by an experienced electrophysiologist into either normal or LAVA categories, extremely noisy signals which did not allow for a reliable classification were discarded. All labels were validated by a different electrophysiologist. Samples of these signals are shown in Figure 3. Table I summarizes the number of electrode locations and bipolar electrograms recorded as well as the proportion of LAVA and normal signals encountered for the EP study of each patient.

\section{Methods: Combined Imaging And Modelling}

We first aim to extract local image features in the vicinity of the location where intracardiac electrograms were recorded and therefore introduce the concept of catheter's sensing range. It refers to the volume of tissue that influences the recording at a particular electroanatomical point (EAP) and it is this region that should be considered when computing image features to describe a particular EAP. Following the recommendations of the experienced electrophysiologist involved in this work, it is represented by a sphere of radius empirically set to $10 \mathrm{~mm}$ [22].

\section{A. Image Feature Extraction}

The work in [21] showed that MR image intensities can be used to discriminate the heterogeneous substrate. Voxels contained inside the sensing range of the catheter were used to compute intensity-based features, including minimal, maximal, mean and standard deviation values. Another feature, defined as the standard deviation over the average intensity in the region, was included. Myocardium thickness was calculated and the scar transmurality was defined as the extent of scar through the entire myocardial thickness.

Texture from medical images provide us with additional information inherent to the underlying tissue. This information has been used in applications as cardiac image indexing and retrieval [24], or to predict vascular events [25]. Grey level co-occurence matrices (GLCM) are matrices of the joint probability of occurrence of a pair of grey values separated by a displacement $d=(d x, d y, d z)$. Haralick features are statistics computed on GLCM that emphasize specific texture properties and have been extensively used in medical image analysis [26]. In our study, the GLCM were computed around the center of the myocardium were the EAP had been projected using a ROI of window size of $11 \times 11 \times 11$ pixels $(\sim 9.4 \times 9.4 \times 9.4 \mathrm{~mm})$. Three distances from the central pixel (1, 2 and 4 pixels), 13 directions and 12 Haralick features were considered, resulting in a 468 element texture feature vector per EAP analyzed. Concatenation of the intensity and texture features yielded a final image-based feature vector of 475 dimensions per electroanatomical point. Furthermore, we assigned a confidence weighting value to the samples during the training phase based on their temporal displacement during EGM recording. This way, image features from EAPs which were less affected by movement were considered more reliable by the classification algorithm. The methods have been more thoroughly described in [22].

\section{B. Model-based Feature Augmentation}

The first step in our model-based feature augmentation scheme is the generation of synthetic intracardiac electrograms, for which we require three main components: a cardiac source model which will be able to describe the depolarization and repolarization activity of the cardiac 
tissue, a tissue model which will be able to account for the differences in electrophysiological properties of the scar and the grey zone and, finally, an electrogram recording model which will describe the electrical activity at the location of a particular catheter.

Cardiac Source Model: Cardiac electrophysiology can be described through a variety of mathematical models [27][30]. More detailed cell-specific models also exist which aim to describe cell-to-cell variability in the cardiac tissue [31], [32]. These models have evolved in the last decades to better represent physiological phenomena [33], e.g. some have been used to study VT or other arrhythmia episodes [34]-[37]. We chose a cardiac electrophysiology model able to represent complex cardiac electrical phenomena while keeping the number and variation of the involved variables tractable: the Mitchell-Schaeffer (MS) [30] model. It has two variables: $u$, the transmembrane potential and $z$, a secondary variable in charge of controlling the repolarisation phase. The model is governed by the following two equations:

$$
\left\{\begin{array}{l}
\partial_{t} u=\operatorname{div}(D \nabla u)+\frac{z u^{2}(1-u)}{\tau_{\text {in }}}-\frac{u}{\tau_{\text {out }}}+J_{\text {stim }}(t) \\
\partial_{t} z=\left\{\begin{array}{lll}
\frac{(1-z)}{\tau_{\text {open }}} & \text { if } u<u_{\text {gate }} \\
\frac{-z}{\tau_{\text {close }}} & \text { if } u>u_{\text {gate }}
\end{array}\right.
\end{array}\right.
$$

Where $D=d \cdot \operatorname{diag}(1, r, r)$ is an anisotropic diffusion tensor that enables the conduction velocity in the fibre direction to be 2.5 times faster than in the transverse plane $R=\frac{1}{2.5^{2}}$. The parameters $\tau_{\text {in }}$ and $\tau_{\text {out }}$ define the depolarisation and repolarisation phases whereas $\tau_{\text {open }}$ and $\tau_{\text {close }}$ are responsible for the action potential duration and the refractory period, based on the change-over voltage $u_{\text {gate }} . J_{\text {stim }}$ is the stimulation current at the pacing location.

The implementation of the Mitchell-Schaeffer model in the SOFA public framework (details described in [38]) was used.

Tissue Model: Imaging data were used to construct a personalized bi-ventricular model, including distributions of scarred and grey zone regions. The MS model parameters were changed in order to account for the differences in electrophysiological properties of the infarcted and border zone tissue with respect to the healthy myocardium. Table II shows the set of parameters used for our personalized simulations. Details of the modifications leading to these parameter values can be found in [23].

TABLE II: Simulation Parameter Values (taken from [23])

\begin{tabular}{lrrr} 
Parameter & Healthy Tissue & Grey Zone & Scar Tissue \\
\hline$\tau_{\text {in }}[\mathrm{ms}]$ & 0.3 & 0.42 & $10 \times 10^{3}$ \\
$\tau_{\text {out }}[\mathrm{ms}]$ & 6 & 8.4 & $10 \times 10^{3}$ \\
$\tau_{\text {close }}[\mathrm{ms}]$ & 150 & 187.5 & 187.5 \\
$\tau_{\text {open }}[\mathrm{ms}]$ & 120 & 120 & 120 \\
$u_{\text {gate }}$ & 0.13 & 0.13 & 0.13 \\
AP Peak Amplitude & 1.0 & 0.7 & 0.7 \\
Conductivity & 4.0 & 0.4 & 0.4
\end{tabular}

EGM recording model: The cardiac source model with the tissue-specific parameters and the electrogram recordings were computed simultaneously using a dipole approach as done in
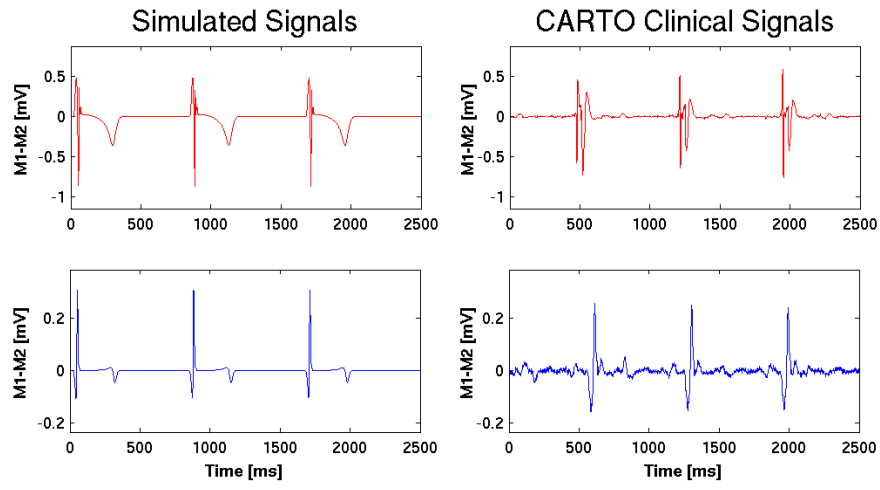

Fig. 5: Sample LAVA (red) and non-LAVA (blue) simulated signals and their clinical counterpart (recorded by the CARTO EP system) are shown. Nevertheless, it is important to note that the blue CARTO signal presents a certain degree of noise, which to the untrained eye might seem as abnormal activations. Also the red CARTO signal presents a steep and high amplitude potential occurring after the QRS complex, a LAVA, which does not happen in the blue signal. This characteristic is well reproduced in the simulated LAVA signal, whereas the simulated blue signal presents a clean depolarization complex and (due to the model characteristics as detailed in [23]) appears noise-free.

[23] and [39]. As in [40], we modelled every myocardium volume element (tetrahedron) as a spatially fixed but time varying current dipole. We define the equivalent current density $\mathbf{j}_{e q}$ as:

$$
\mathbf{j}_{e q}=-\sigma^{i} \nabla v
$$

where $\mathbf{j}_{e q}$ is a current dipole moment per unit of volume and the local dipole moment $\mathbf{p}$ in the volume $V$ writes as $\mathbf{p}=$ $\int_{V} \mathbf{j}_{e q} d V$. According to the volume conductor theory [41], the electric potential at a distance $R$ in a homogeneous volume conductor of conductivity $\sigma_{b}$ is:

$$
\Psi(R)=\frac{1}{4 \pi \sigma_{b}} \int_{V} \mathbf{j}_{e q} \cdot \nabla\left(\frac{1}{R}\right) d V
$$

We model the moving propagation front as a dipole field. The infinitesimal dipole moment of the volume $d V_{X}$ located at position $X$ is defined as $\mathbf{p}_{X}=\mathbf{j}_{e q, X} d V_{X}=-\sigma_{X}^{i} \nabla v_{X} d V_{X}$. As we use linear tetrahedra in the FEM discretization of the myocardium, the potential $v$ is linear and $\nabla v$ is constant over the tetrahedron. We get the following formulation of the dipole moment of the charge in the volume $V_{H}$ of tetrahedron $H$ of the myocardial mesh: $\mathbf{p}_{H}=-\sigma_{H}^{i} \nabla v_{H} V_{H}$ The gradient of the electric potential $\nabla v_{H}$ for a tetrahedron $H$ is estimated using the node positions $X_{H}^{k}$ and the shape vectors $\overrightarrow{D_{H}^{k}}$ of the tetrahedron $H$ [42]:

$$
\overrightarrow{D_{H}^{k}}=\frac{s}{V_{H}}\left(X_{H}^{k \oplus 2}-X_{H}^{k \oplus 1}\right) \times\left(X_{H}^{k \oplus 3}-X_{H}^{k \oplus 1}\right)
$$

where $s=1$ for $k=2,4, s=-1$ for $k=1,3$, and $k \oplus l=$ $(k-1+l) \bmod 3+1$. The gradient of the electric potential in 
the tetrahedron $H$ is then computed from the potentials $v\left(X_{H}^{k}\right)$ at the nodes $X_{H}^{k}$ as:

$$
\nabla v_{H}=\sum_{k=1}^{4} v\left(X_{H}^{k}\right) \overrightarrow{D_{H}^{k}}
$$

From Equation 3, the contribution $\Psi_{H}$ of the tetrahedron $H$ to the potential field calculated at position $X_{T}$ is:

$$
\Psi_{H}\left(X_{T}\right)=\frac{1}{4 \pi \sigma_{T}} \frac{\sigma_{H}^{i} V_{H}\left(\nabla v_{H} \cdot \overrightarrow{H T}\right)}{\|\overrightarrow{H T}\|^{3}}
$$

with $\overrightarrow{H T}$ the vector from centre of the tetrahedron $H$ to the torso electrode location $T$. Finally, we sum over the whole mesh to get the potential field at $X_{T}$.

The recording catheter used in the clinical environment has four electrodes named M1, M2, M3 and M4 from the distal to the proximal. The locations obtained from the CARTO system during the recording of the clinical electrograms were used to simulate unipolar electrograms. Therefore, a single electrode location will be associated with both a clinical unipolar signal and a simulated one. Then, in both the clinical and the simulation scenario, two bipolar recordings are generated from these unipolar measurements: M1-M2 and M3-M4. Because the far-field signal is assumed to be similar for both unipolar recordings, it is largely filtered out in bipolar measurements and they are useful to study local activities. It is on these bipolar measurements that LAVA signals can be identified. Sample signals for simulated intracardiac electrograms and their clinical CARTO counterpart are show in Figure 5.

Extraction of Augmented Features. Feature extraction was performed on the distal-most bipolar simulated EGMs. Eight model-based features, shown in Figure 6, were extracted from the simulated electrograms: signal range, number of inflection points, signal energy, dominant frequency, mean slope, fractionation index and minimum and maximum signal values. Details concerning EGM feature extraction are discussed in [23]. These features were concatenated to the ones obtained from the image. Similarly as in the image-based learning scheme, a confidence weighting based on temporal displacement during EGM recording was incorporated in the training phase.

A full summary of the features used is shown in Table III.

TABLE III: Augmented Feature Summary

Feature Description

\section{Image Features}

Intensity-based

Myocardial thickness

Scar transmurality

Texture-based

Simulation Features

Amplitude-based

Energy/frequency

Clinical EGM description (fractionation)
Dimensions
5

1

1

468

3

2

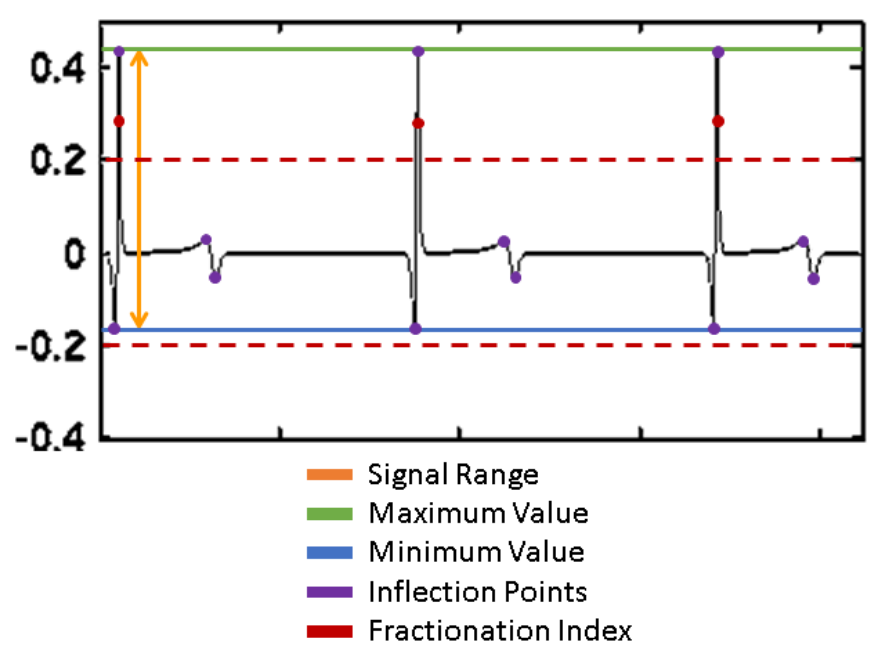

Fig. 6: Features obtained through the use of a model-based feature augmentation scheme.

\section{Uncertainty Assessment}

We derived a principled analysis of confidence impact on classification. Inspired by cost-sensitive learning, we formulate the problem as samples $(x, y, c)$ drawn from a distribution D on a domain $X \times Y \times C$ with $\mathrm{X}$ being the input feature space, $\mathrm{Y}$ corresponding to the binary output class and $\mathrm{C}$ to the confidence associated with each sample. We aim to learn a classifier $h: X \rightarrow Y$ which minimizes the new expected classification error $\mathbf{E}$ :

$$
E(x, y, c \sim D)[c I(h(x) \neq y)]
$$

Where $I(\cdot)$ is the indicator function that has a value of 1 in case the argument is true and 0 otherwise. Using the Translation Theorem 2.1 in [43] we can compute and draw samples from a distribution $D^{\prime}$ such that the optimal error rate classifiers for $D^{\prime}$ are optimal cost minimizers for data drawn from $\mathrm{D}$. We derive how this modifies the training using weights to simulate the expectation of finite data $E(x, y \sim D)[I(x, y)]$ as:

$$
E(x, y \sim D)[f(x, y)]=\frac{1}{\sum c} \sum c I(x, y)
$$

equivalent to importance sampling for $D^{\prime}$ using distribution $\mathrm{D}$, so the modified expectation is an unbiased Monte Carlo estimate of the expectation with respect to $D^{\prime}$ [43]. In random forests, the node split criterion is information gain:

$$
I G=H(S)-\sum_{i=1,2} \frac{\left|S^{i}\right|}{|S|} H\left(S^{i}\right)
$$

with $|S|$ being the number of samples in a node before split, $\left|S^{i}\right|$ being the number of samples of each children node and $H(S)$ the Shannon entropy:

$$
H(S)=-\sum_{c \in C} p(c) \log (p(c))
$$

where $\mathrm{p}(\mathrm{c})$ is calculated as normalized empirical histogram of labels corresponding to the training points in $S, p(c)=\frac{\left|S^{i}\right|}{|S|}$. 
Using weighted instances, $p(c)$ is replaced by $p_{w}(c)$, which has the following formulation:

$$
\begin{gathered}
p_{w}^{i}(c)=\frac{\sum \text { Weights of samples of class c in node } \mathrm{i}}{\sum \text { Weights of samples in node } \mathrm{i}} \\
p_{w}^{i}(c)=\frac{\sum_{S_{c}} W^{i}}{\sum_{S} W^{i}}
\end{gathered}
$$

This yields a sample weighted formulation of the information gain that can be written as:

$$
I G=H(W)-\sum_{i=1,2} \frac{\sum_{S} W^{i}}{\sum_{S} W} H\left(W^{i}\right)
$$

where $\mathrm{W}$ are sample weights at the parent node and $W^{i}$ are sample weights that have been passed to each child node. $H(W)$ is given by:

$$
H(W)=-\sum_{c \in C} p_{w}(c) \log \left(p_{w}(c)\right)
$$

Due to breathing and cardiac motions, the recording catheter is displaced throughout the 2.5 seconds of recording time. Magnitudes varying significantly among electro-anatomical points (EAP) so, intuitively, features from EAP with smaller displacement are considered to be more reliable. The covariance of the position matrix is obtained and an ellipsoid with radii $2 \sqrt{\operatorname{diag}(P)}$ is generated, where $\mathrm{P}$ is the matrix containing the eigenvalues along the main diagonal. The major ellipsoid radius, $T D=\max (2 \sqrt{\operatorname{diag}(P)})$, defines the temporal displacement. Each EAP is assigned a confidence value by linearly scaling the temporal displacement to a weight parameter with range of $[0.5,1]$ where 0.5 corresponds to the lowest confidence and 1 to the highest. We first presented this formulation to strengthen our methodological approach in [22].

\section{Machine Learning Framework}

Similarly to what was presented in [22], we used a random forest [44] classification framework with a five-fold nested cross-validation scheme [45]. The use of nested crossvalidation, with a parameter-tuning inner loop and an outer loop for performance estimation, avoided an optimistic bias introduction into generalization estimate [45]. The number of trees in the classifier, their respective depth and the maximum number of features used were optimized for PPV performance using the Python implementation from the scikit-learn library [46].

\section{Evaluation Metrics}

In order to be clinically useful, the results of the classification algorithms were projected onto the endocardial surface mesh to create confidence maps for potential RF ablation targets. The study in [47] reports an average endocardial area of $3.5 \mathrm{~cm}^{2}$ for RFA lesions. Therefore, the endocardial surface was divided into regions of area $1.7 \mathrm{~cm}^{2}$, two times smaller than the average RFA lesions. Figure 7 shows a sample endocardial surface mesh partition.

EAP were projected to their closest endocardial surface region along with their prediction and confidence results. Also,

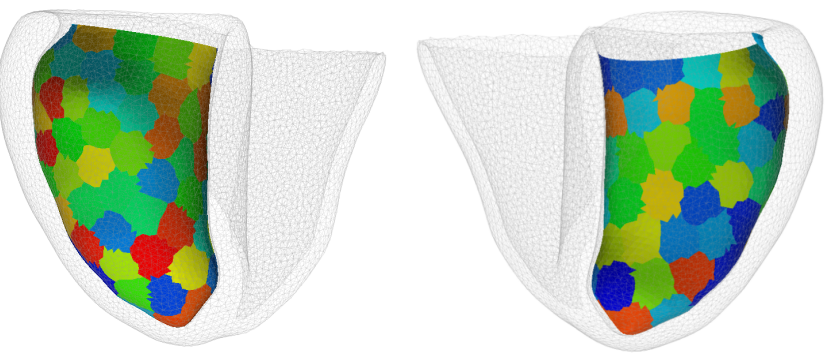

Fig. 7: (Left) Anterior and (Right) posterior resulting regions for classification result display after endocardial surface division.

a region was considered LAVA if at least one of the EAP projected onto it was labelled as such. The justification behind this is given by the physical constraints of RFA: the ablation of a LAVA site will affect neighbouring tissue within the extension of the RFA lesion size, even if they are considered non-LAVA inducing.

After projection of the classification results onto the regions in the surface mesh and its comparison with the ground truth, a confusion matrix was generated and the following statistics were computed: accuracy, sensitivity and positive predictive value $(\mathrm{PPV})$.

\section{RESUlTS AND DisCUSSION}

\section{A. Classification Results}

By applying the presented method on 5 patients, we obtained the classification results detailed in Table IV. For illustrative purposes, prediction maps from Patient 1 are shown on Figure 8.

TABLE IV: Classification Statistics

\begin{tabular}{lccccc}
\hline Learning & Patient & Accuracy & Sensitivity & Specificity & PPV \\
\hline Image & P1 & 94.0 & 73.3 & 98.5 & 91.6 \\
& P2 & 92.6 & 33.3 & 100 & 100 \\
& P3 & 91.8 & 39.0 & 99.0 & 90.0 \\
& P4 & 93.7 & 11.0 & 100 & 100 \\
& P5 & 92.5 & 10.0 & 100 & 100 \\
& Mean & $\mathbf{9 2 . 9}$ & $\mathbf{3 3 . 2}$ & $\mathbf{9 9 . 4}$ & $\mathbf{9 6 . 4}$ \\
\hline Simulation & P1 & 89.2 & 73.3 & 92.7 & 68.7 \\
& P2 & 95.4 & 66.6 & 98.0 & 88.0 \\
& P3 & 92.6 & 78.0 & 98.0 & 90.0 \\
& P4 & 99.2 & 89.0 & 100 & 100 \\
& P5 & 95.7 & 60.0 & 100 & 100 \\
& Mean & $\mathbf{9 4 . 4}$ & $\mathbf{7 3 . 2}$ & $\mathbf{9 7 . 8}$ & $\mathbf{8 9 . 4}$ \\
\hline After Feature & P1 & 95.2 & 80.0 & 98.5 & 92.3 \\
Augmentation & P2 & 96.3 & 66.6 & 100 & 100 \\
& P3 & 96.2 & 87.0 & 98.0 & 83.0 \\
& P4 & 99.2 & 89.0 & 100 & 100 \\
& P5 & 98.9 & 90.0 & 100 & 100 \\
& Mean & $\mathbf{9 7 . 2}$ & $\mathbf{8 2 . 4}$ & $\mathbf{9 9 . 2}$ & $\mathbf{9 5 . 0}$ \\
\hline
\end{tabular}

- Results from image-based learning. As can be seen from Table IV, the algorithm has an overall accuracy of 92.9\% across the five patients, with a LAVA-specificity of $99.4 \%$ and a LAVA-PPV of $96.4 \%$. This means that when a region is classified as LAVA, in general, the 

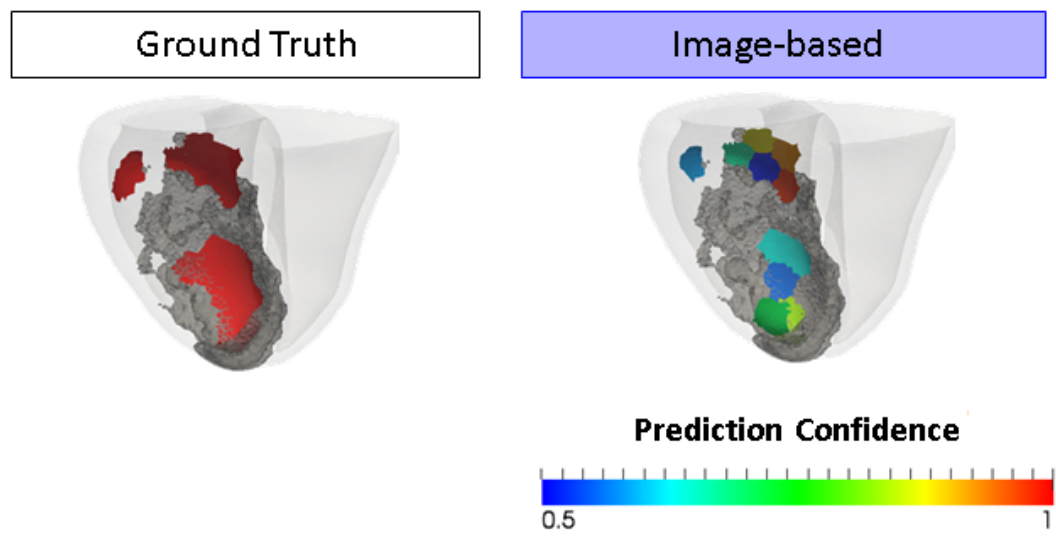

with Augmented Features

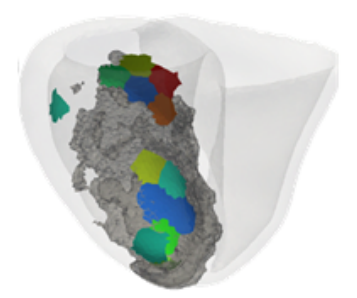

Fig. 8: (Top) LAVA regions from ground truth of Patient 1 and predicted LAVA regions with confidence level color-coding for image-based features and image + model-based augmented features.
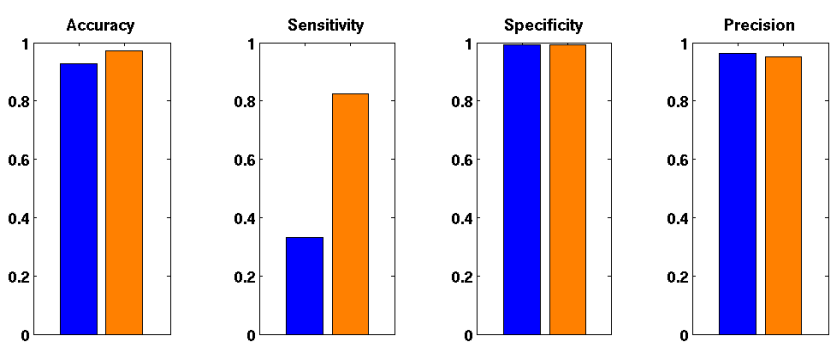

Fig. 9: Classification statistics summary on five patients using image-only features (blue) and image plus augmented modelbased features (orange).

algorithm is quite reliable. Nevertheless, there is a severe discrepancy in the scores of LAVA-sensitivity across patients, being as high as $73 \%$ as in Patient 1 or as low as $10 \%$ as in Patient 5.

- Results from simulation-based learning. Although the results from using only simulation-based features are slightly lower in terms of LAVA-specificity (97.8\%) and PPV (89.4\%) than those obtained through image-based learning, they remain high. The overall accuracy of the algorithm across the five patients is of $94.4 \%$, but more importantly, the sensitivity increased by almost $40 \%$.

- Results after model-based feature augmentation. Significantly higher scores in accuracy (97.2\%) and even more in LAVA-sensitivity (82.4\%) scores were achieved after incorporating feature augmentation while yielding high values for LAVA-specificity (99.2\%) and LAVA-PPV (95.0\%) across the five patients. Therefore, it can be said that this scheme is able to boost both the accuracy and LAVA-sensitivity obtained from purely image-based methods while slightly compromising on the specificity and PPV.

From the previous results it an be seen that the use of an augmented feature set containing both imaging and simulation- based features outperforms the overall results from either method alone. The deficiencies of the simulation-based method in PPV score are compensated by the high scores of the imagebased method and, more importantly, the synergy between both methods allows for an overall increase of LAVA-sensitivity across patients.

It is also interesting to note that the patient with the lowest scores has a scar-to-healthy tissue ratio of 0.25 , the highest in our patient cohort. Similarly, the patient with the smallest scar-to-healthy tissue ratio had the highest sensitivity in our database.

On the other hand, the algorithm's performance is not correlated to the number of LAVA in the dataset, as Patients 2 and 5, with the two lowest percentages, present both the lowest and highest sensitivity scores when using an augmented feature set.

\section{B. Preliminary Results on Inter-patient Learning}

Given the large variability in etiologies, image quality and catheter recording noise level, using a classifier learned from a different patient is challenging. Nevertheless, we performed preliminary inter-patient classification experiments using the two patients in the database whose mean feature vector resembled the most. The mean feature vector of a patient was obtained by averaging the feature values for all its EAP. The Euclidean distance of the current test patient's feature vector to each of the patients in the database was computed and the learning algorithm was trained using only the features from the closest subject. Figure 10 shows the distance between the feature vectors of all the patients in the database.

Furthermore, the threshold in classification probability was optimized. Traditional random forest binary classification algorithms label a given sample based on the probability distribution of both classes. The label assigned is that of the class with the highest probability, therefore, even the slightest increase from a 50\% probability would result in a hard label. Nevertheless, for the purposes of our particular application, we wish to leverage our clinical understanding of the problem to explore the use of a more clinically-suitable threshold. In this 


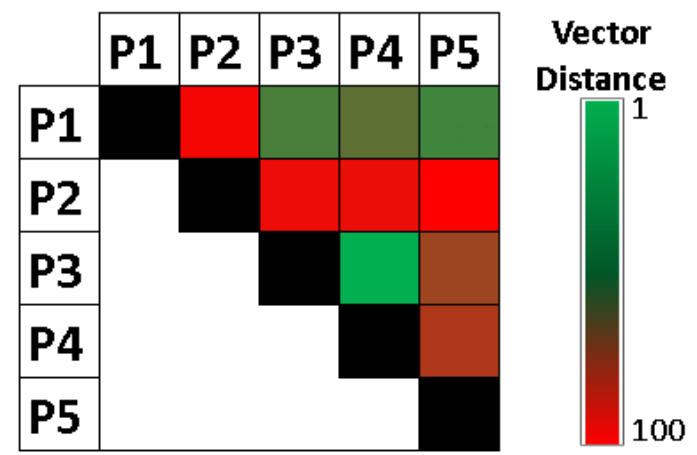

Fig. 10: Grid depicting the normalized Euclidean distance between the mean feature vectors of all patients. Patients 3 and 4 have the most similar vector while Patient 2 is shown to have the most dissimilar one to the rest of the database.

context, as the output of our algorithm would be providing the clinician with a map of regions that would be potential ablation targets, it is preferable to minimize the number of false negatives (FN) in the classification. This is because during the intervention, a clinician could always assess the tissue marked as a target prior to performing an ablation. While having a considerable percentage of false positives (FP) would signify that the clinician would have to manually verify them before ablation, it is preferable than having an algorithm yielding a considerable number of false negatives. This would fail to mark as ablation candidates regions in the myocardial tissue that are LAVA-inducing, and because the clinician would not be driven to analyse these regions, it could compromise the success of the therapy. Therefore, we gradually increased the threshold values for classification to find an optimal threshold $t$. That is to say, a region should have at least a confidence $t$ of it belonging to a non-LAVA inducing region, before classifying it as so. This will primarily impact the sensitivity of the LAVAinducing regions while increasing the PPV of the classification.

These preliminary experiments were only performed using the two patients with highest resemblance in their feature vectors (Patient 3 and 4), which are also the two patients with the most similar value of scar-to-healthy tissue ratio in our patient cohort. As can be seen from Table V, the average classification scores were of $96.8 \%$ accuracy, $95.9 \%$ specificity and $89.6 \%$ PPV. While very preliminary, we consider these results open the doors to a proof of concept for an entirely non-invasive assessment of the presence of LAVA inducing regions in a patient.

TABLE V: Inter-patient Classification Statistics. (trained on the closest patient, given in parenthesis with the normalized Euclidean distance using optimal classification threshold)

\begin{tabular}{lccccc}
\hline Learning & Patient & Accuracy & Sensitivity & Specificity & PPV \\
\hline Closest & P4 (P3, 7.40) & 98.4 & 100.0 & 98.3 & 83.3 \\
$(t=0.60)$ & P3 (P4, 7.40) & 95.1 & 91.7 & 95.6 & 75.9 \\
& Mean & $\mathbf{9 6 . 8}$ & $\mathbf{9 5 . 9}$ & $\mathbf{9 6 . 9}$ & $\mathbf{7 9 . 6}$ \\
\hline
\end{tabular}

\section{Discussion}

From these experiences, it can be seen that model-based feature augmentation improves the classification scores obtained by using only image-based features. Particularly it increases the algorithm's sensitivity to abnormal electrograms by incorporating physiological knowledge into the data-driven learning system.

We recognize that one of the limitations of this work is the reduced patient cohort due to the challenges in acquiring such comprehensive data for the study. These challenges include, but are not limited to, the need for both cardiac MRI and electroanatomical mapping, as well as detailed labelling of this data by experts, which is a time-consuming process (late-enhancement MR segmentation is still not automatised, and we have between 800 and 4800 recorded electrodes per patient to label). Furthermore, most patients undergoing RFA for VT were not suitable for this work as the MR imaging study could not be performed due to the presence of an implantable cardioverter-defibrillator (ICD). Nevertheless, despite this limitation, we believe this work is an encouraging proof of concept of the use of biophysical modeling for feature augmentation in a machine learning framework, in order to improve the accuracy of ablation point identification.

In the results section, it was described how Patient 2, with a scar-to-healthy tissue ratio of 0.25 , had the lowest performances, while Patients 3, 4, and 5, with a scar-to-healthy tissue ratio lower than 0.10 , had better overall performances. This might lead us to believe that one of the limitations of our method is its capability of extrapolating to patients with significantly large scars, and that it would be best suited for patients with a relatively small scar-to-healthy tissue ratio. While the current state of our modeling framework allows us to modify the electrophysiological parameters of healthy, grey zone and scarred tissue, the electrophysiological properties of large sections of damaged tissue might become less homogeneous, giving rise to greater differences between the clinically and simulated electrogram features.

While preliminary, we also showed that inter-patient analysis can yield encouraging results by training on a small cohort (the closest patient) and leveraging on the understanding of the clinical problem further drives the algorithm to perform better for our clinical application.

The authors consider that a line of future work should focus on the construction of larger databases. One way to achieve this is to use synthetic data with realistic characteristics similar to what was performed in [48]. These samples could include realistic myocardial infarctions with different grey zone to scar core ratios, sizes, shapes and locations and intracardiac electrograms could be computed from them. After considerably increasing the size of our database (with both real and synthetic samples) we could consider more sophisticated learning algorithms, such as deep learning, and evaluate them in a clinical study.

If this larger study were to be successful, the authors see the inclusion of an approach like the one proposed in this paper to be feasible from a technical standpoint. For a new incoming patient, only a non-invasive imaging study would be needed 
in order to generate a personalized model of the patient's cardiac anatomy, including differentiation between the healthy and scarred regions of the heart. Next, electrophysiological modelling and imaging features could be extracted and compared to an existing database. The simulation and analysis time could be shortened to meet clinical needs, especially with the current advances in hardware, which could allow for rapid parallel processing. The analysis of this pre-intervention data would allow for an improvement in the accuracy of RFA target identification, reduction of the intervention time and an increase in the success rates of RFA.

\section{CONCLUSION}

We presented the use of a biophysical model for feature augmentation to improve the performance of machine learning, for the prediction of RFA target identification defined by the presence of LAVA. The image data was obtained from delayed-enhanced MR imaging while feature augmentation was performed through the use of a personalized imagebased model for the simulation of intracardiac electrograms. First, the classification performance was assessed using only image-based features. Next, an augmented feature vector was used for classification. Their performances were evaluated for five patients in an intra-patient manner. We found that using a model-based feature augmentation scheme had better performance when assessed using four classification metrics.

Finally, a preliminary inter-patient learning experiment was put in place. Because only a reduced and highly heterogeneous database was available, we restricted the training set to the single closest patient in the database, as opposed to using all the patients contained in it, and we adjusted the classification threshold as opposed to using the traditional 0.5 threshold. These results open up possibilities to introduce physiological knowledge through biophysical modelling into machine learning approaches, in order to improve the prediction results.

\section{ACKNOWLEDGMENT}

Part of this work was funded by the European Research Council through the ERC Advanced Grant MedYMA 2011291080 (on Biophysical Modeling and Analysis of Dynamic Medical Images).

\section{REFERENCES}

[1] M. Nichols, N. Townsend, P. Scarborough, and M. Rayner, "Cardiovascular disease in europe 2014: epidemiological update," European Heart Journal, p. ehu299, 2014.

[2] E. M. Aliot, W. G. Stevenson, J. M. Almendral-Garrote, F. Bogun, C. H. Calkins, E. Delacretaz, P. Della Bella, G. Hindricks, P. Jaïs, and M. E. Josephson, "Ehra/hrs expert consensus on catheter ablation of ventricular arrhythmias developed in a partnership with the european heart rhythm association (ehra), a registered branch of the european society of cardiology (esc), and the heart rhythm society (hrs); in collaboration with the american college of cardiology (acc) and the american heart association (aha)," Europace, vol. 11, no. 6, pp. 771$817,2009$.

[3] F. E. Marchlinski, D. J. Callans, C. D. Gottlieb, and E. Zado, "Linear ablation lesions for control of unmappable ventricular tachycardia in patients with ischemic and nonischemic cardiomyopathy," Circulation, vol. 101, no. 11, pp. 1288-1296, 2000.
[4] H. Kottkamp, U. Wetzel, P. Schirdewahn, A. Dorszewski, J.-H. Gerdsli, C. Carbucicchio, R. Kobza, and G. Hindricks, "Catheter ablation of ventricular tachycardia in remote myocardial infarction," Journal of Cardiovascular Electrophysiology, vol. 14, no. 7, pp. 675-681, 2003.

[5] K. Soejima, M. Suzuki, W. H. Maisel, C. B. Brunckhorst, E. Delacretaz, L. Blier, S. Tung, H. Khan, and W. G. Stevenson, "Catheter ablation in patients with multiple and unstable ventricular tachycardias after myocardial infarction short ablation lines guided by reentry circuit isthmuses and sinus rhythm mapping," Circulation, vol. 104, no. 6, pp. 664-669, 2001.

[6] K. Soejima, W. G. Stevenson, W. H. Maisel, J. L. Sapp, and L. M. Epstein, "Electrically unexcitable scar mapping based on pacing threshold for identification of the reentry circuit isthmus feasibility for guiding ventricular tachycardia ablation," Circulation, vol. 106, no. 13, pp. 16781683, 2002.

[7] H. Ashikaga, T. Sasano, J. Dong, M. M. Zviman, R. Evers, B. Hopenfeld, V. Castro, R. H. Helm, T. Dickfeld, S. Nazarian et al., "Magnetic resonance-based anatomical analysis of scar-related ventricular tachycardia," Circulation research, vol. 101, no. 9, pp. 939-947, 2007.

[8] T. Dickfeld, P. Lei, V. Dilsizian, J. Jeudy, J. Dong, A. Voudouris, R. Peters, M. Saba, R. Shekhar, and S. Shorofsky, "Integration of threedimensional scar maps for ventricular tachycardia ablation with positron emission tomography-computed tomography," JACC: Cardiovascular Imaging, vol. 1, no. 1, pp. 73-82, 2008.

[9] C. De Chillou, L. Groben, I. Magnin-Poull, M. Andronache, M. M. Abbas, N. Zhang, A. Abdelaal, S. Ammar, J.-M. Sellal, and J. Schwartz, "Localizing the critical isthmus of postinfarct ventricular tachycardia: the value of pace-mapping during sinus rhythm," Heart Rhythm, vol. 11, no. 2, pp. 175-181, 2014.

[10] F. Bogun, E. Good, S. Reich, D. Elmouchi, P. Igic, K. Lemola, D. Tschopp, K. Jongnarangsin, H. Oral, and A. Chugh, "Isolated potentials during sinus rhythm and pace-mapping within scars as guides for ablation of post-infarction ventricular tachycardia," Journal of the American College of Cardiology, vol. 47, no. 10, pp. 2013-2019, 2006.

[11] R. N. Ghanem, P. Jia, C. Ramanathan, K. Ryu, A. Markowitz, and Y. Rudy, "Noninvasive electrocardiographic imaging (ecgi): comparison to intraoperative mapping in patients," Heart Rhythm, vol. 2, no. 4, pp. 339-354, 2005.

[12] G. Li and B. He, "Localization of the site of origin of cardiac activation by means of a heart-model-based electrocardiographic imaging approach," IEEE transactions on biomedical engineering, vol. 48, no. 6 , pp. 660-669, 2001.

[13] B. He, G. Li, and X. Zhang, "Noninvasive three-dimensional activation time imaging of ventricular excitation by means of a heart-excitation model," Physics in medicine and biology, vol. 47, no. 22, p. 4063, 2002.

[14] X. Zhang, I. Ramachandra, Z. Liu, B. Muneer, S. M. Pogwizd, and B. He, "Noninvasive three-dimensional electrocardiographic imaging of ventricular activation sequence," American Journal of Physiology-Heart and Circulatory Physiology, vol. 289, no. 6, pp. H2724-H2732, 2005.

[15] C. Liu, N. D. Skadsberg, S. E. Ahlberg, C. M. Swingen, P. A. Iaizzo, and $\mathrm{B}$. He, "Estimation of global ventricular activation sequences by noninvasive three-dimensional electrical imaging: Validation studies in a swine model during pacing," Journal of cardiovascular electrophysiology, vol. 19, no. 5, pp. 535-540, 2008.

[16] C. Liu, M. D. Eggen, C. M. Swingen, P. A. Iaizzo, and B. He, "Noninvasive mapping of transmural potentials during activation in swine hearts from body surface electrocardiograms," IEEE transactions on medical imaging, vol. 31, no. 9, pp. 1777-1785, 2012.

[17] L. Wang, O. A. Gharbia, B. M. Horáček, and J. L. Sapp, "Noninvasive epicardial and endocardial electrocardiographic imaging of scar-related ventricular tachycardia," Journal of electrocardiology, vol. 49, no. 6, pp. 887-893, 2016.

[18] L. Di Biase, J. D. Burkhardt, D. Lakkireddy, C. Carbucicchio, S. Mohanty, P. Mohanty, C. Trivedi, P. Santangeli, R. Bai, G. Forleo et al., "Ablation of stable vts versus substrate ablation in ischemic cardiomyopathy: the vista randomized multicenter trial," Journal of the American College of Cardiology, vol. 66, no. 25, pp. 2872-2882, 2015.

[19] P. Jaïs, P. Maury, P. Khairy, F. Sacher, I. Nault, Y. Komatsu, M. Hocini, A. Forclaz, A. S. Jadidi, and R. Weerasooryia, "Elimination of local abnormal ventricular activities a new end point for substrate modification in patients with scar-related ventricular tachycardia," Circulation, vol. 125, no. 18, pp. 2184-2196, 2012.

[20] K. H. Schuleri, M. Centola, R. T. George, L. C. Amado, K. S. Evers, K. Kitagawa, A. L. Vavere, R. Evers, J. M. Hare, and C. Cox, "Characterization of peri-infarct zone heterogeneity by contrast-enhanced multidetector computed tomography: a comparison with magnetic resonance 
imaging," Journal of the American College of Cardiology, vol. 53, no. 18, pp. 1699-1707, 2009.

[21] E. Perez-David, Á. Arenal, J. L. Rubio-Guivernau, R. Del Castillo, L. Atea, E. Arbelo, E. Caballero, V. Celorrio, T. Datino, and E. Gonzalez-Torrecilla, "Noninvasive identification of ventricular tachycardia-related conducting channels using contrast-enhanced magnetic resonance imaging in patients with chronic myocardial infarction: comparison of signal intensity scar mapping and endocardial voltage mapping," Journal of the American College of Cardiology, vol. 57, no. 2, pp. 184-194, 2011.

[22] R. Cabrera-Lozoya, J. Margeta, L. Le Folgoc, Y. Komatsu, B. Benjamin, J. Relan, H. Cochet, M. Haissaguerre, P. Jais, N. Ayache, and M. Sermesant, "Confidence-based training for clinical data uncertainty in imagebased prediction of cardiac ablation targets," in bigMCV Workshop on the Medical Image Computing and Computer Assisted Intervention Conference - MICCAI 2014, 2014.

[23] R. Cabrera-Lozoya, B. Berte, H. Cochet, P. Jaïs, N. Ayache, and M. Sermesant, "Image-based Biophysical Simulation of Intracardiac Abnormal Ventricular Electrograms," IEEE Transactions on Biomedical Engineering, vol. PP, no. 99, 2016.

[24] T. Glatard, J. Montagnat, and I. E. Magnin, "Texture based medical image indexing and retrieval: application to cardiac imaging," in Proceedings of the 6th ACM SIGMM international workshop on Multimedia information retrieval. ACM, 2004, pp. 135-142.

[25] A. van Engelen, T. Wannarong, G. Parraga, W. J. Niessen, A. Fenster, J. D. Spence, and M. de Bruijne, "Three-dimensional carotid ultrasound plaque texture predicts vascular events," Stroke, vol. 45, no. 9, pp. 2695 2701, 2014.

[26] L. Tesar, D. Smutek, A. Shimizu, and H. Kobatake, "3d extension of haralick texture features for medical image analysis," in Signal Processing, Pattern Recognition and Applications (SPPRA) 2007 proceedings of the fourth conference on International Association of Science and Technology for Development (IASTED) international conference, 2007, pp. 350-355.

[27] R. R. Aliev and A. V. Panfilov, "A simple two-variable model of cardiac excitation," Chaos, Solitons \& Fractals, vol. 7, no. 3, pp. 293-301, Mar. 1996. [Online]. Available: http://www.sciencedirect.com/science/ article/B6TJ4-3VTC35G-C/1/90f6ad80f6ac98f60a2f9410729ad20c

[28] P. N. K. ten Tusscher, D. Noble and A. Panfilov, "A model for human ventricular tissue," American Journal of Physiology - Heart and Circulatory Physiology, 2004.

[29] F. Fenton and A. Karma, "Erratum: "vortex dynamics in threedimensional continuous myocardium with fiber rotation: Filament instability and fibrillation" [chaos 8, 20-47 (1998)]." Chaos, vol. 8, no. 4, p. 879, Dec. 1998. [Online]. Available: http://dx.doi.org/10.1063/ 1.166374

[30] C. Mitchell and D. Schaeffer., "A two-current model for the dynamics of cardiac membrane." Bulletin of Mathematical Biology, 2003.

[31] M. Zaniboni, A. E. Pollard, L. Yang, and K. W. Spitzer, "Beat-to-beat repolarization variability in ventricular myocytes and its suppression by electrical coupling," American Journal of Physiology-Heart and Circulatory Physiology, vol. 278, no. 3, pp. H677-H687, 2000.

[32] A. X. Sarkar, D. J. Christini, and E. A. Sobie, "Exploiting mathematical models to illuminate electrophysiological variability between individuals," The Journal of physiology, vol. 590, no. 11, pp. 2555-2567, 2012.

[33] A. E. Pollard, "From myocardial cell models to action potential propagation," Journal of electrocardiology, vol. 36, pp. 43-49, 2003.

[34] M. P. Nash and A. V. Panfilov, "Electromechanical model of excitable tissue to study reentrant cardiac arrhythmias," Progress in biophysics and molecular biology, vol. 85, no. 2, pp. 501-522, 2004.

[35] I. V. Kazbanov, R. H. Clayton, M. P. Nash, C. P. Bradley, D. J. Paterson, M. P. Hayward, P. Taggart, and A. V. Panfilov, "Effect of global cardiac ischemia on human ventricular fibrillation: Insights from a multi-scale mechanistic model of the human heart," 2014.

[36] J. Relan, P. Chinchapatnam, M. Sermesant, K. Rhode, M. Ginks, H. Delingette, C. A. Rinaldi, R. Razavi, and N. Ayache, "Coupled personalization of cardiac electrophysiology models for prediction of ischaemic ventricular tachycardia," Journal of the Royal Society Interface Focus, vol. 1, no. 3, pp. 396-407, 2011. [Online]. Available: http://www.inria.fr/sophia/asclepios/Publications/Jatin.Relan/ RSFS-2010-0041_Manuscript.pdf

[37] K. S. McDowell, S. Zahid, F. Vadakkumpadan, J. Blauer, R. S. MacLeod, and N. A. Trayanova, "Virtual electrophysiological study of atrial fibrillation in fibrotic remodeling," PloS one, vol. 10, no. 2, p. e0117110, 2015.
[38] H. Talbot, S. Marchesseau, C. Duriez, M. Sermesant, S. Cotin, and $\mathrm{H}$. Delingette, "Towards an interactive electromechanical model of the heart," Interface Focus, vol. 3, no. 2, 2013.

[39] S. Giffard-Roisin, T. Jackson, L. Fovargue, J. Lee, H. Delingette, R. Razavi, N. Ayache, and M. Sermesant, "Non-Invasive Personalisation of a Cardiac Electrophysiology Model from Body Surface Potential Mapping," IEEE Transactions on Biomedical Engineering, 2016.

[40] C. E. Chávez, N. Zemzemi, Y. Coudière, F. Alonso-Atienza, and D. Alvarez, "Inverse problem of electrocardiography: Estimating the location of cardiac ischemia in a 3d realistic geometry," in International Conference on Functional Imaging and Modeling of the Heart. Springer, 2015, pp. 393-401.

[41] J. Malmivuo and R. Plonsey, Bioelectromagnetism: principles and applications of bioelectric and biomagnetic fields. Oxford university press, 1995.

[42] H. Delingette and N. Ayache, "Soft tissue modeling for surgery simulation," Handbook of Numerical Analysis, vol. 12, pp. 453-550, 2004.

[43] B. Zadrozny, J. Langford, and N. Abe, "Cost-sensitive learning by costproportionate example weighting," in Data Mining, 2003. International Conference on Data Mining (ICDM) 2003. Third IEEE International Conference on. IEEE, 2003, pp. 435-442.

[44] A. Criminisi, J. Shotton, and E. Konukoglu, "Decision forests for classification, regression, density estimation, manifold learning and semi-supervised learning," Microsoft Research Cambridge, Tech. Rep. MSRTR-2011-114, vol. 5, no. 6, p. 12, 2011.

[45] M. Ruschhaupt, W. Huber, A. Poustka, and U. Mansmann, "A compendium to ensure computational reproducibility in high-dimensional classification tasks," Statistical Applications in Genetics and Molecular Biology, vol. 3, no. 1, 2004.

[46] F. Pedregosa, G. Varoquaux, A. Gramfort, V. Michel, B. Thirion, O. Grisel, M. Blondel, P. Prettenhofer, R. Weiss, and V. Dubourg, "Scikit-learn: Machine learning in python," The Journal of Machine Learning Research, vol. 12, pp. 2825-2830, 2011.

[47] K. Ilg, T. S. Baman, S. K. Gupta, S. Swanson, E. Good, A. Chugh, K. Jongnarangsin, F. Pelosi, T. Crawford, and H. Oral, "Assessment of radiofrequency ablation lesions by $\mathrm{cmr}$ imaging after ablation of idiopathic ventricular arrhythmias," Journal of the American College of Cardiology: Cardiovascular Imaging, vol. 3, no. 3, pp. 278-285, 2010

[48] N. Duchateau, M. De Craene, P. Allain, E. Saloux, and M. Sermesant, "Infarct localization from myocardial deformation: Prediction and uncertainty quantification by regression from a low-dimensional space," IEEE transactions on medical imaging, vol. 35, no. 10, pp. 2340-2352, 2016. 